\title{
Bone turnover markers to explain changes in lumbar spine BMD with abaloparatide and teriparatide: results from ACTIVE
}

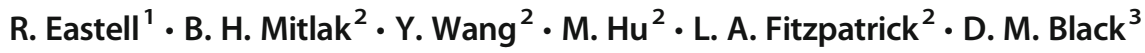

Received: 29 August 2018 / Accepted: 5 December 2018 / Published online: 11 January 2019

(C) The Author(s) 2019

\begin{abstract}
Summary Early PINP changes correlate with 18-month lumbar spine BMD changes and the correlation was greater with abaloparatide versus teriparatide. The uncoupling index was similar between the two agents.

Introduction We evaluated the relationship between early PINP changes and subsequent changes in spine BMD following abaloparatide and teriparatide treatments. We also explored the use of an "uncoupling index" (UI), the balance between bone formation and bone resorption, which we hypothesised would be similar in response to these treatment groups.

Methods Blood samples were taken for measurement of bone turnover markers (BTMs) s-PINP and s-CTX at baseline, 1, 3, 6 , 12, and 18 months from 189 abaloparatide patients and 227 teriparatide patients randomly selected from all participants who completed the study. BMD was measured by DXA at baseline, 6, 12, and 18 months. Correlations were calculated between log ratio of BTMs from baseline to 3 months and percent change from baseline in BMD at 18 months. A UI was calculated using log transformation and subtraction of the standard deviate for s-CTX from the standard deviate for s-PINP for each patient.

Results Early BTM changes were associated with subsequent BMD changes for both treatments. Pearson correlations for the log ratio of PINP over baseline at 3 months and BMD percent change from baseline at 18 months were larger $(P<0.0001)$ with abaloparatide $(r=0.561)$ than teriparatide $(r=0.198)$. The mean UI at 1 month was greater for abaloparatide versus teriparatide (1.743 and 1.493, respectively; $P=0.03$ ) but was similar at 3 months or later time points.

Conclusions Early s-PINP changes correlate with percentage change in lumbar spine BMD 18 months after treatment with both abaloparatide and teriparatide, though the correlation with abaloparatide was greater. The UI was similar between abaloparatide and teriparatide suggesting that the balance between formation and resorption markers was similar.
\end{abstract}

Keywords Abaloparatide $\cdot$ Bone mineral density $\cdot$ Bone turnover markers · Procollagen type I N propeptide $\cdot$ Teriparatide Uncoupling index

\section{Introduction}

Abaloparatide [1] is a synthetic peptide analogue of the human parathyroid hormone-related protein used in the treatment of postmenopausal osteoporosis in women at high risk for fracture. Abaloparatide binds to the parathyroid hormone 1 receptor, similar to teriparatide or PTH 1-34. Recent in vitro studies

R. Eastell

r.eastell@sheffield.ac.uk

1 Mellanby Centre for Bone Research, University of Sheffield, Sheffield, UK

2 Radius Health, Inc., Waltham, MA, USA

3 University of California, San Francisco, CA, USA indicate that there are at least two stable, high-affinity PTHR1 conformations, $\mathrm{R}^{0}$ and $\mathrm{RG}$. Binding to $\mathrm{R}^{0}$ results in prolonged signalling responses in cells and prolonged calcemic responses in animals, whereas selective binding to RG results in more transient responses. Abaloparatide has greater selectivity for the RG conformation than teriparatide and is thought to promote more transient signalling which results in an anabolic effect with less resorption [2].

Bone turnover markers (BTMs) can be used to monitor the effects of osteoporosis treatment. The International Osteoporosis Foundation has proposed that the reference marker for bone formation should be procollagen type I N propeptide (s-PINP) and serum carboxy-terminal cross-linking telopeptide of type I collagen (s-CTX) for bone resorption [3].

s-PINP has proven to be the most sensitive marker for detecting the response of bone turnover to teriparatide [4]. S- 
PINP increases within 3 days of starting this treatment [5] and reaches a peak after 3 to 6 months of treatment [6]. The early change in s-PINP correlates with the later change in BMD whether the treatment was with teriparatide $[7,8]$ or PTH 184 [9]. The relationship between early changes in biochemical markers of bone turnover and subsequent BMD response to abaloparatide has not been examined.

The ACTIVE Study compared abaloparatide with blinded placebo and with open-label teriparatide over 18 months [10]. In ACTIVE, the increase in lumbar spine BMD was similar for abaloparatide compared to teriparatide and the increase in total hip BMD was greater for abaloparatide, yet the increase in the bone formation marker s-PINP was lower with abaloparatide than teriparatide. The increase in the bone resorption marker s-CTX was also lower, raising the question of whether the relative increase of formation and resorption might differ and explain the differences in the pattern of BMD changes. The relative changes in bone resorption and formation can be estimated mathematically by calculating the mean and standard deviation of the relevant markers and subtracting resorption from formation [11]. This approach has been used during therapy of glucocorticoid-induced osteoporosis with teriparatide [12].

If the PINP is increased more by teriparatide than by abaloparatide, then why is the increase in BMD at the spine at 18 months similar for the two drugs and the increase in BMD at the total hip at 18 months different? Perhaps this could be explained by studying the relationship between the change in bone formation and bone resorption markers quantitatively using an 'uncoupling index'.

\section{Materials and methods}

\section{Subjects}

The Abaloparatide Comparator Trial In Vertebral Endpoints (ACTIVE) was a phase 3, double-blind, randomised controlled trial conducted at 28 sites in 10 countries [10]. Postmenopausal women with bone mineral density (BMD) T-score $\leq-2.5$ and $>-5.0$ at the lumbar spine or femoral neck and radiological evidence 2 mild or 1 moderate lumbar or thoracic vertebral fracture or history of low-trauma nonvertebral fracture within the past 5 years were eligible. Postmenopausal women ( $>65$ years) meeting the fracture criteria and having a T-score between -2.0 and $>-5.0$, or not meeting the fracture criteria and having a $\mathrm{T}$-score between -3.0 and $>-5.0$, could enrol. The abaloparatide $80 \mu$ group $(n=824)$ and placebo group $(n=821)$ received masked pens that were identical in appearance, while the teriparatide $20 \mu \mathrm{g}$ group $(n=818)$ was open-label and patients received commercial teriparatide pens.

\section{Sample collection and BTM analyses}

In the phase 3 ACTIVE, blood samples were taken under fasting conditions at baseline and 1, 3, 6, 12, and 18 months and measured for BTMs among a subset of patients who were randomly selected from all sites based on a simple random selection procedure in a treatment-blinded fashion to reach a target sample size of 600 (approximately 200 patients per treatment group). Patients who did not complete the ACTIVE trial were excluded from the random selection.

Validated bioanalytical assays for BTMs used the automated Roche Elecsys platform. Biomarker measurements were performed at the Nordic Synarc Research Labs (Denmark) using a COBAS E411 automated analyser (Roche Diagnostics, Germany) according to the manufacturer's instructions. The s-PINP intra-assay and inter-assay precision was reported as $<2.9 \%$ and $<3.7 \%$, respectively, with a lower limit of quantification (LLOQ) and upper limit of quantification (ULOQ) of $5 \mathrm{ng} / \mathrm{ml}$ and $1200 \mathrm{ng} / \mathrm{ml}$, respectively. The sCTX intra-assay and inter-assay precision were reported as $<$ $3.2 \%$ and $<3.4 \%$, respectively, with an LLOQ and ULOQ of 0.010 and $6.0 \mathrm{ng} / \mathrm{ml}$, respectively. We measured $1,25(\mathrm{OH})_{2} \mathrm{D}$ by EIA (Immunodiagnostic Systems, Boldon, UK).

\section{Statistical analysis}

The correlation of change from baseline in concentration of sPINP and s-CTX at 1, 3, 6, 12, and 18 months was compared with percent change from baseline in lumbar spine BMD at 18 months using Spearman's rank correlation. Because the distribution of BTM data was skewed, we also evaluated the correlation of the log ratio of s-PINP and s-CTX over baseline with percent change from baseline in lumbar spine BMD at 18 months using Pearson's correlation coefficients. For example, if the PINP was 60 at follow-up and 50 at baseline, then we would take the ratio, 1.2, and then its natural logarithm, 0.182 . Scatter plots between log ratio of s-PINP at 3 months and lumbar spine BMD percent change at 18 months were presented with linear regression lines for abaloparatide and teriparatide. To compare between treatments, Pearson correlation coefficients were assessed using the Z-score test after Fischer's Z transformation. $T$ test was used to assess the difference in slopes of the regression lines between the two treatment groups.

The 'uncoupling index' (UI) was calculated as the Z-score of $\log$ s-PINP minus the Z-score of log s-CTX. The Z-score standardised marker values were calculated by taking the difference between each subject's bone turnover marker and the mean of all subjects' bone turnover markers at baseline divided by the associated standard deviation.

All tests were 2-sided and performed at a significance level of 0.05 , with no adjustment for multiple comparisons. Analyses used observed data without imputing for missing 
values. The statistical software used was SAS version 9.4 (SAS Institute, Cary, NC).

\section{Results}

Among the 600 randomly selected patients in the BTM subset, 184 received placebo, 189 received abaloparatide, and 227 received teriparatide. The baseline demographics were similar between the abaloparatide and the teriparatide groups (Table 1). They were also similar to the patients treated in the main trial with abaloparatide $(n=824)$ and teriparatide $(n=818)[10]$.

The serum concentrations of s-PINP and s-CTX during treatment and the uncoupling index (UI) are shown in Fig. 1. Both abaloparatide and teriparatide increased sPINP and s-CTX concentrations as reported by Miller [10]. The pattern of increase was similar for the two agents during the first 3 months of treatment, but thereafter, s-PINP and s-CTX concentrations were greater for teriparatide than abaloparatide. Despite these differences, the UI was generally similar for abaloparatide and teriparatide during 18 months of treatment. The mean UI was moderately greater for abaloparatide than teriparatide at 1 month (1.743 vs 1.493 , respectively; $P=0.03)$, but not at 3 months (Fig. 1, Table 2) or at later time points.

Table 1 Baseline demographics and characteristics

\begin{tabular}{lll}
\hline & Abaloparatide & Teriparatide \\
\hline Variable & $(N=189)$ & $(N=227)$ \\
Age (years), Mean (SD) & $68.6(6.5)$ & $68.4(6.6)$ \\
Race $n(\%)$ & & \\
White & $154(81.5)$ & $182(80.2)$ \\
Asian & $28(14.8)$ & $35(15.4)$ \\
Black or African American & $6(3.2)$ & $6(2.6)$ \\
Other & $1(0.5)$ & $4(1.8)$ \\
Hispanic or Latino & $40(21.2)$ & $48(21.1)$ \\
BMI (kg/m $\left.{ }^{2}\right)$, mean (SD) & $25.0(3.2)$ & $25.4(3.5)$ \\
Prevalent vertebral fracture, $n(\%)$ & $45(23.8)$ & $59(26.0)$ \\
No prior fracture, $n(\%)$ & $66(34.9)$ & $88(38.8)$ \\
Bone mineral density, T-score, mean (SD) & \\
Lumbar spine & $-2.9(0.88)$ & $-2.8(0.85)$ \\
Femoral neck & $-2.1(0.63)$ & $-2.1(0.65)$ \\
Total hip & $-1.8(0.77)$ & $-1.8(0.75)$ \\
Bone turnover markers, mean (SD) & & \\
s-PINP (ng/mL) & $54.5(20.7)$ & $53.5(20.6)$ \\
s-CTX (ng/mL) & $0.49(0.19)$ & $0.48(0.20)$ \\
\hline
\end{tabular}

$B M I$, body mass index; BTM, bone turnover markers; $s$-CTX, serum carboxy-terminal cross-linking telopeptide of type I collagen; $S D$, standard deviation; $s$-PINP, procollagen type I N propeptide
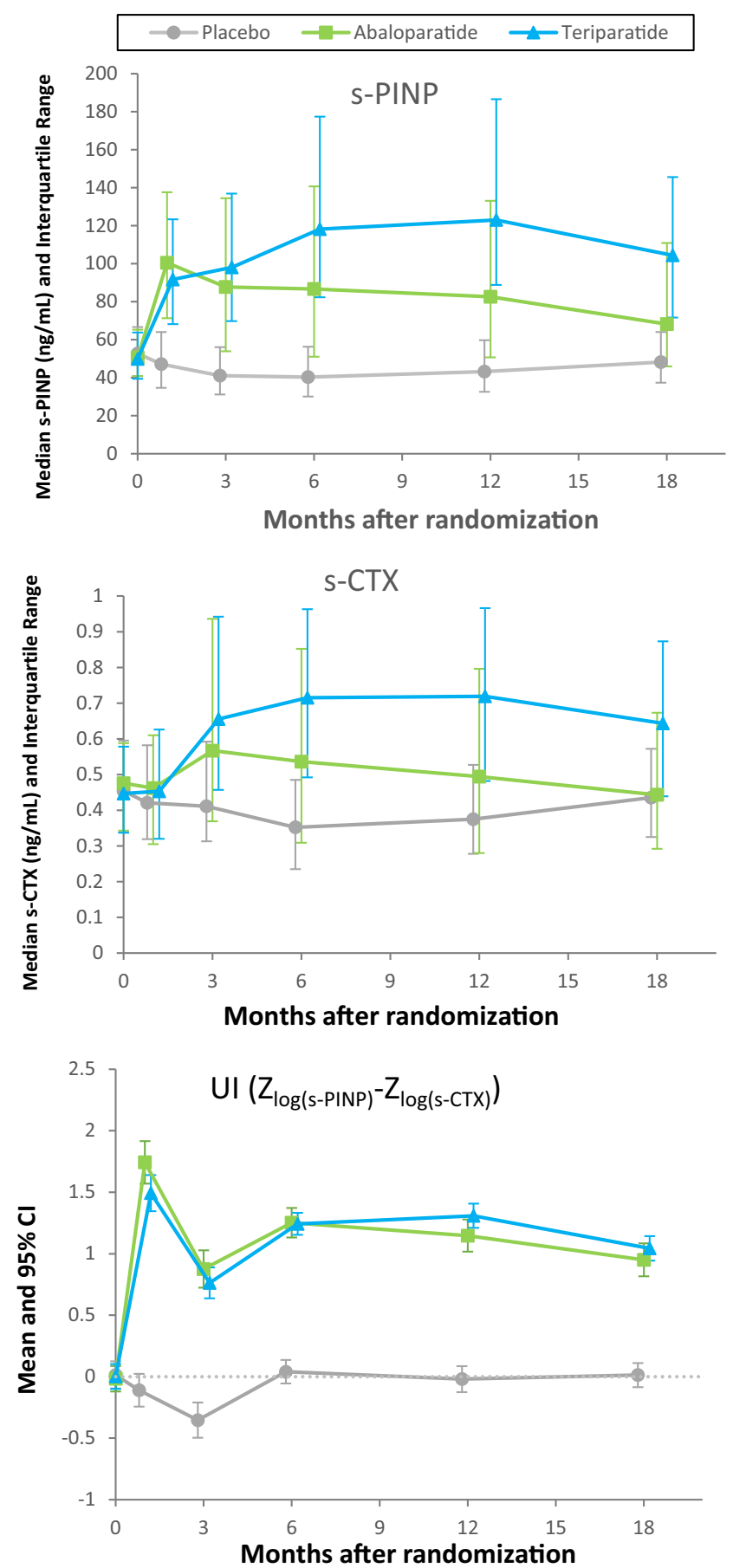

Fig. 1 Median (interquartile range) of s-PINP, s-CTX and mean (95\% CI) of uncoupling index over time during treatment with placebo, abaloparatide, or teriparatide over 18 months

For patients included in this BTM analysis, BMD increased at the lumbar spine, total hip, and femoral neck with abaloparatide and teriparatide similar to the overall cohort [10]. After 18 months of treatment, the percentage change in lumbar spine BMD was similar between agents, but the percentage increase in total hip and femoral neck BMD was 
Table 2 Summary of bone turnover markers and the uncoupling index at 3 months

\begin{tabular}{|c|c|c|c|}
\hline & $\begin{array}{l}\text { Abaloparatide } \\
N=189\end{array}$ & $\begin{array}{l}\text { Teriparatide } \\
N=227\end{array}$ & $P$ value \\
\hline \multicolumn{4}{|c|}{ s-PINP at baseline (ng/mL) } \\
\hline Median (IQR) & $50.6(40.8,65.3)$ & $49.9(39.4,63.8)$ & 0.435 \\
\hline \multicolumn{4}{|c|}{$\Delta \mathrm{s}-\mathrm{PINP}$ at month $3(\mathrm{ng} / \mathrm{mL})$} \\
\hline Median (IQR) & $28.9(3.2,81.8)^{*}$ & $47.8(20.9,76.8)$ & 0.005 \\
\hline \multicolumn{4}{|c|}{ Log ratio of s-PINP at month 3 over baseline } \\
\hline Mean (SD) & $0.56(0.66)^{*}$ & $0.68(0.45)$ & 0.016 \\
\hline \multicolumn{4}{|c|}{ s-CTX at baseline (ng/mL) } \\
\hline Median (IQR) & $0.48(0.34,0.59)$ & $0.45(0.34,0.58)$ & 0.361 \\
\hline \multicolumn{4}{|c|}{$\Delta \mathrm{s}-\mathrm{CTX}$ at month $3(\mathrm{ng} / \mathrm{mL})$} \\
\hline Median (IQR) & $0.07(-0.04,0.33)^{*}$ & $0.20(0.07,0.39)$ & $<0.0001$ \\
\hline \multicolumn{4}{|c|}{ Log ratio of s-CTX at month 3 over baseline } \\
\hline Mean (SD) & $0.23(0.54)^{*}$ & $0.41(0.40)$ & $<0.0001$ \\
\hline \multicolumn{4}{|c|}{$\mathrm{UI}\left(\mathrm{Z}_{\log (\mathrm{s}-\mathrm{PINP})}-\mathrm{Z}_{\log (\mathrm{s}-\mathrm{CTX})}\right)$ at month 3} \\
\hline & $0.88(1.05)^{*}$ & & 0.259 \\
\hline Mean (SD) & & $0.76(0.96)$ & \\
\hline
\end{tabular}

$* N=187$

$I Q R$, interquartile range; $s$-CTX, serum carboxy-terminal cross-linking telopeptide of type I collagen; $S D$, standard deviation; $s$-PINP, procollagen type I $\mathrm{N}$ propeptide

significantly greater for abaloparatide as compared to teriparatide (Fig. 2).

There was a significant correlation between early change in s-PINP and subsequent increases in BMD for both therapies. The strongest correlations between s-PINP and BMD were observed between 3 and 6 months; the 3month time point was considered most clinically useful for providing early feedback and is described in more detail. The relationship between the log ratio of s-PINP at 3 months and percentage change in lumbar spine at 18 months for abaloparatide was $r=0.561 \quad(P<0.0001)$ and $r=0.198(P=0.003)$ for teriparatide; the correlation coefficient for abaloparatide was significantly larger than for teriparatide $(P<0.0001)$ (Table 3, Fig. 3). The removal of an outlying point (ratio of about 20 ) only had a small effect on this correlation for the teriparatide group (it changed from an $r$ value of 0.198 to 0.215 ). The slope of the regression line between $\log$ ratio of s-PINP at 3 months and increase in lumbar spine BMD at 18 months was greater for abaloparatide (slope $=5.96$ ) than for teriparatide (slope $=2.39$ ) (Fig. $3 ; P=0.0005)$ indicating that a similar early increase in s-PINP was associated with a greater increase in subsequent lumbar spine BMD. A significant correlation between the 3-month $\log$ ratio of s-PINP and total hip BMD was also observed for abaloparatide $(r=0.273 ; P<0.001)$ but not for teriparatide (Table 3 ).

There was also a significant correlation between early change in s-CTX and subsequent increases in BMD. The relationship between change in the log ratio of s-CTX at 3 months and percentage change in lumbar spine BMD at 18 months for abaloparatide was $r=0.451(P<0.0001)$ and $r=0.042(P=0.53)$ for teriparatide; the correlation coefficient for abaloparatide was significantly larger than for teriparatide $(P<0.0001)$ (Table 3, Fig. 3). A significant correlation between the 3-month log ratio of s-CTX and total hip BMD was also observed for abaloparatide $(r=0.166 ; P=0.023)$, but not for teriparatide (Table 3).

We also examined the relationship between the UI and subsequent increases in BMD. There was a significant correlation between the 3-month UI for abaloparatide and lumbar spine BMD after 18 months $(r=0.247$; $P<0.001)$, but not for teriparatide $(r=0.115 ; P=\mathrm{NS}$; $P=$ NS for the comparison between UI for abaloparatide versus teriparatide) (Table 3). A similar pattern was observed with total hip BMD (Table 3).

The changes in BTM could be influenced by changes in $1,25(\mathrm{OH})_{2} \mathrm{D}$. However, the mean increases in $1,25(\mathrm{OH})_{2} \mathrm{D}$ at 1,6 , and 18 months for treatment with abaloparatide were 26 , 16 , and $13 \%$ and for teriparatide were 27,18 , and $15 \%$.
Fig. 2 Mean change (95\% CI) in lumbar spine and total hip BMD over time during treatment with placebo, abaloparatide, or teriparatide over 18 months for patients included in the BTM analysis

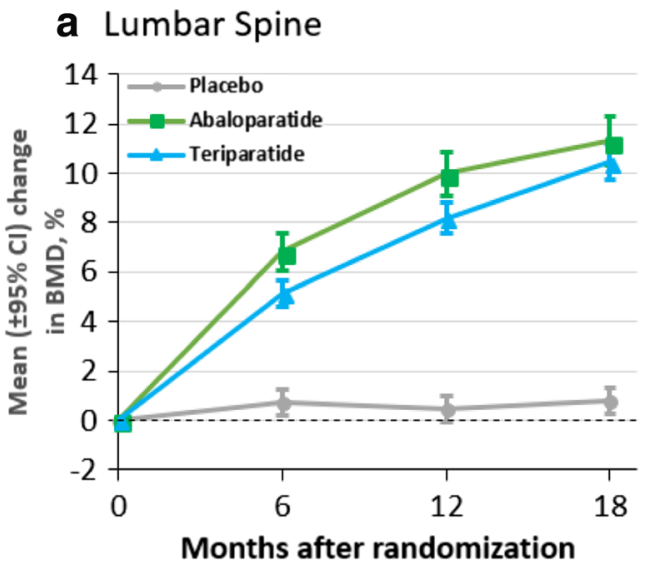

b Total hip

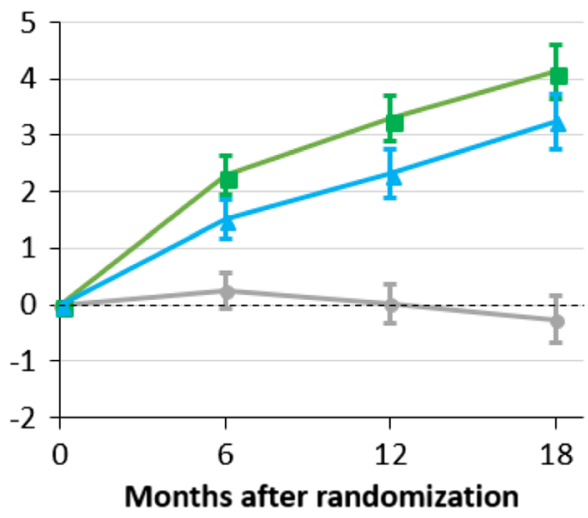


Table 3 Correlation of bone turnover marker at 3 months and percent change in bone mineral density at 18 months

\begin{tabular}{|c|c|c|c|c|}
\hline & \multicolumn{2}{|c|}{ Lumbar spine BMD } & \multicolumn{2}{|c|}{ Total hip BMD } \\
\hline & $\mathrm{ABL}$ & TPTD & $\mathrm{ABL}$ & TPTD \\
\hline \multicolumn{5}{|c|}{ Baseline s-PINP } \\
\hline $\mathrm{n}$ & 188 & 227 & 189 & 225 \\
\hline Corr. Coef. & 0.088 & 0.274 & 0.155 & 0.249 \\
\hline$P$ value & 0.229 & $<0.0001$ & 0.034 & $<0.001$ \\
\hline \multicolumn{5}{|c|}{$\Delta$ s-PINP at 3 months } \\
\hline$n$ & 186 & 227 & 187 & 225 \\
\hline Corr. Coef. & 0.536 & 0.264 & 0.280 & 0.043 \\
\hline$P$ value & $<0.0001$ & $<0.001$ & $<0.001$ & 0.519 \\
\hline \multicolumn{5}{|c|}{ Log ratio of s-PINP at 3 months over baseline } \\
\hline$n$ & 186 & 227 & 187 & 225 \\
\hline Corr. Coef. & 0.561 & 0.198 & 0.273 & -0.054 \\
\hline$P$ value & $<0.0001$ & 0.003 & $<0.001$ & 0.422 \\
\hline \multicolumn{5}{|c|}{$P$ value of ABL Corr Coef vs TPTD Corr Coef } \\
\hline & & $<0.0001$ & & $<0.001$ \\
\hline \multicolumn{5}{|c|}{ Baseline s-CTX } \\
\hline$n$ & 188 & 227 & 189 & 225 \\
\hline Corr Coef & 0.175 & 0.309 & 0.128 & 0.237 \\
\hline$P$ value & 0.016 & $<0.0001$ & 0.079 & $<0.001$ \\
\hline \multicolumn{5}{|c|}{$\Delta \mathrm{s}-\mathrm{CTX}$ at 3 months } \\
\hline$n$ & 186 & 227 & 187 & 225 \\
\hline Corr Coef & 0.439 & 0.156 & 0.139 & 0.031 \\
\hline$P$ value & $<0.0001$ & 0.019 & 0.058 & 0.640 \\
\hline \multicolumn{5}{|c|}{ Log ratio of s-CTX at 3 months over baseline } \\
\hline$n$ & 186 & 227 & 187 & 225 \\
\hline Corr Coef & 0.451 & 0.042 & 0.166 & -0.111 \\
\hline$P$ value & $<0.0001$ & 0.530 & 0.023 & 0.096 \\
\hline \multicolumn{5}{|c|}{$P$ value of ABL Corr Coef vs TPTD Corr Coef } \\
\hline & & $<0.0001$ & & 0.005 \\
\hline \multicolumn{5}{|c|}{$\mathrm{UI}\left(\mathrm{Z}_{\log (\mathrm{s}-\mathrm{PINP})}-\mathrm{Z}_{\log (\mathrm{s}-\mathrm{CTX})}\right)$ at month 3} \\
\hline$n$ & 186 & 227 & 187 & 225 \\
\hline Corr Coef & 0.247 & 0.115 & 0.257 & 0.032 \\
\hline$P$ value & $<0.001$ & 0.083 & $<0.001$ & 0.635 \\
\hline \multicolumn{5}{|c|}{$P$ value of ABL Corr Coef vs TPTD Corr Coef } \\
\hline & & 0.171 & & 0.021 \\
\hline
\end{tabular}

$A B L$, abaloparatide; $B M D$, bone mineral density; Corr Coef, correlation coefficient; $s$-CTX, serum carboxy-terminal cross-linking telopeptide of type I collagen; $s$-PINP, procollagen type I N propeptide; TPTD, teriparatide

\section{Discussion}

In this study, we have tried to explain why two anabolic drugs differ in the increase in the bone formation marker s-PINP, yet had similar lumbar spine BMD increase. We think this might be explained by an alteration in the relationship between change in s-PINP and BMD and by the similarity in the increase in s-PINP relative to s-CTX.

We concluded this because we had examined the relationship between changes in BTMs and changes in BMD and found that this relationship differed for abaloparatide and teriparatide, with the slope being steeper for abaloparatide. It is unknown as to why the relationship is different for these two drugs. It may be that the relative effects on modelling and remodelling differ, but this would require a comparative study of bone histomorphometry.

An alternative, yet speculative, explanation is that regions of the skeleton differ in their responses to the treatments. BTMs reflect metabolism occurring within the entire skeleton; hence, if there were particularly potent effects of teriparatide on the skull [13], this would result in greater increases in BTMs, but not greater anabolic effects on BMD at the lumbar spine or total hip.

We hypothesised that the change in balance between formation and resorption early in treatment would be more predictive of 18 -month change in lumbar spine BMD than a single marker of either formation or resorption alone. Thus, we calculated the UI, which increased in response to both anabolic agents. Such a finding has been reported previously for teriparatide in glucocorticoid-induced osteoporosis, with a value of about 2 at 3 months [12]. However, the UI was calculated differently in the current study as we addressed the skewed distribution of the BTMs by logarithmic transformation. We had used a similar approach recently to demonstrate an increase in bone turnover associated with bone loss in postmenopausal women [14]. The early difference in UI between treatments at 1 month, which was no longer apparent at 3 months or later time points, could help explain why lumbar spine BMD increased more quickly with abaloparatide but was similar to teriparatide after 18 months; however, this does not fully explain why there was a greater increase in proximal femur BMD with abaloparatide.

Bone histology has been reported for the ACTIVE study [15]. It would be helpful in the future to reconstruct the bone remodelling cycle in order to calculate 'bone balance from resorption depth and mean wall thickness' as this is the histological equivalent of 'uncoupling index'.

The strengths of our study are the large sample size given that we are studying surrogates, the continuous outcomes, and the inclusion of both teriparatide and abaloparatide in the same study, with patients randomly assigned to treatment. Also, the BTM and BMD were measured using state-of-the-art techniques, and we analysed the data while taking account of the skewed distribution of BTMs. The weaknesses are that teriparatide was given as an open-label treatment and there is an insufficient number of fractures to compare the relationship between changes in bone turnover markers and fracture. 
Fig. 3 The relationship between change in s-PINP or s-CTX at 3 months (expressed as a log ratio) and change in lumbar spine BMD at 18 months. The $r$ alues for abaloparatide were greater than those of teriparatide $(p<0.0001)$
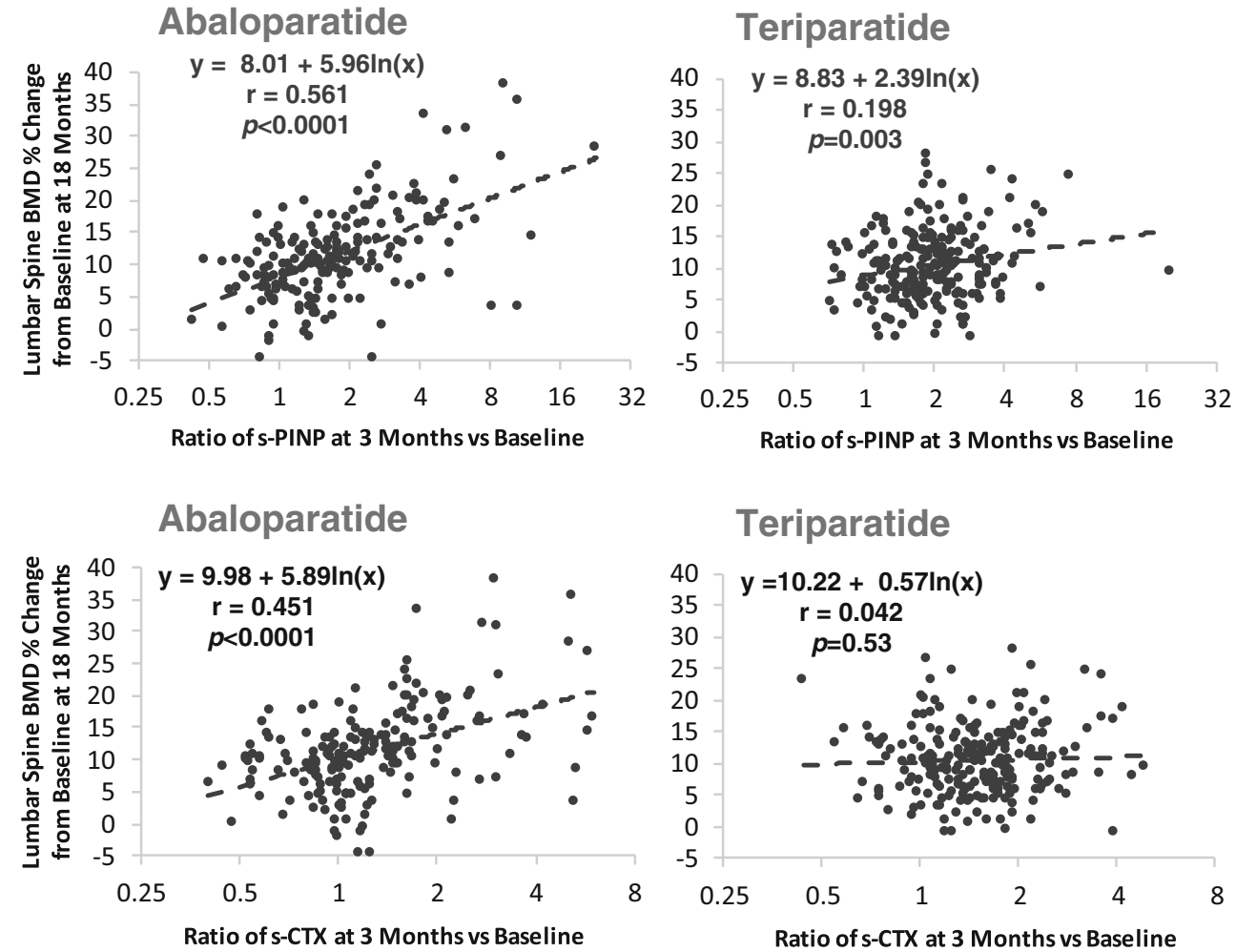

In conclusion, early changes in s-PINP correlate with the percentage change in lumbar spine BMD after 18 months of treatment with both abaloparatide and teriparatide, although the correlation with abaloparatide was greater. The UI was generally similar between abaloparatide and teriparatide, supporting that while absolute levels of s-PINP and s-CTX were lower with abaloparatide than teriparatide, the balance between the formation and resorption marker was similar.

Funding information This study was funded by Radius Health Inc.

\section{Compliance with ethical standards}

Conflicts of interest RE reports grants and personal fees from Amgen; grants from AstraZeneca; grants and personal fees from Alexion; grants, personal fees, and non-financial support from Immunodiagnostic Systems; grants, personal fees, and non-financial support from Roche; personal fees from Lilly; personal fees from D3 Biomedical Science Institutes; personal fees from GSK Nutrition; and travel support from Radius Health; BHM, MH, and LAF YW are employees of and hold stocks in Radius Health Inc.; DMB has been a consultant to Radius Health, Inc. and an adviser to Merck and has performed data analyses for Asahi-Kasei.

Open Access This article is distributed under the terms of the Creative Commons Attribution-NonCommercial 4.0 International License (http:// creativecommons.org/licenses/by-nc/4.0/), which permits any noncommercial use, distribution, and reproduction in any medium, provided you give appropriate credit to the original author(s) and the source, provide a link to the Creative Commons license, and indicate if changes were made.
Publisher's Note Springer Nature remains neutral with regard to jurisdictional claims in published maps and institutional affiliations.

\section{References}

1. Shirley M (2017) Abaloparatide: first global approval. Drugs 77: 1363-1368

2. Hattersley G, Dean T, Corbin BA, Bahar H, Gardella TJ (2016) Binding selectivity of abaloparatide for PTH-type-1-receptor conformations and effects on downstream signaling. Endocrinology 157:141-149

3. Vasikaran S, Eastell R, Bruyere O, Foldes AJ, Garnero P, Griesmacher A, McClung M, Morris HA, Silverman S, Trenti T, Wahl DA, Cooper C, Kanis JA, IOF-IFCC Bone Marker Standards Working Group (2011) Markers of bone turnover for the prediction of fracture risk and monitoring of osteoporosis treatment: a need for international reference standards. Osteoporos Int 22:391-420

4. Eastell R, Krege J, Chen P, Glass E, Reginster J (2006) Development of an algorithm for using PINP to monitor treatment of patients with teriparatide. Curr Med Res Opin 22:61-66

5. Glover SJ, Eastell R, McCloskey EV, Rogers A, Garnero P, Lowery J, Belleli R, Wright TM, John MR (2009) Rapid and robust response of biochemical markers of bone formation to teriparatide therapy. Bone 45:1053-1058

6. Tsujimoto M, Chen P, Miyauchi A, Sowa H, Krege JH (2011) PINP as an aid for monitoring patients treated with teriparatide. Bone 48 : 798-803

7. Blumsohn A, Marin F, Nickelsen T, Brixen K, Sigurdsson G, Gonzalez de la Vera J, Boonen S, Liu-Léage S, Barker C, Eastell R, EUROFORS Study Group (2011) Early changes in biochemical markers of bone turnover and their relationship with bone mineral 
density changes after 24 months of treatment with teriparatide. Osteoporos Int 22:1935-1946

8. Chen P, Satterwhite JH, Licata AA, Lewiecki EM, Sipos AA, Misurski DM, Wagman RB (2005) Early changes in biochemical markers of bone formation predict BMD response to teriparatide in postmenopausal women with osteoporosis. J Bone Miner Res 20: 962-970

9. Bauer DC, Garnero P, Bilezikian JP, Greenspan SL, Ensrud KE, Rosen CJ, Palermo L, Black DM (2006) Short-term changes in bone turnover markers and bone mineral density response to parathyroid hormone in postmenopausal women with osteoporosis. J Clin Endocrinol Metab 91:1370-1375

10. Miller PD, Hattersley G, Riis BJ, Williams GC, Lau E, Russo LA, Alexandersen P, Zerbini CA, Hu MY, Harris AG, Fitzpatrick LA, Cosman F, Christiansen C, Study Investigators ACTIVE (2016) Effect of abaloparatide vs placebo on new vertebral fractures in postmenopausal women with osteoporosis: a randomized clinical trial. JAMA 316:722-733
11. Eastell R, Robins SP, Colwell T, Assiri AM, Riggs BL, Russell RG (1993) Evaluation of bone turnover in type I osteoporosis using biochemical markers specific for both bone formation and bone resorption. Osteoporos Int 3:255-260

12. Lane NE, Sanchez S, Genant HK, Jenkins DK, Arnaud CD (2000) Short-term increases in bone turnover markers predict parathyroid hormone-induced spinal bone mineral density gains in postmenopausal women with glucocorticoid-induced osteoporosis. Osteoporos Int 11:434-442

13. Paggiosi MA, Yang L, Blackwell D, Walsh JS, McCloskey E, Peel N, Eastell R (2018) Teriparatide treatment exerts differential effects on the central and peripheral skeleton: results from the MOAT study. Osteoporos Int March 10 [online ahead of print]

14. Gossiel F, Altaher H, Reid DM, Roux C, Felsenberg D, Glüer CC, Eastell R (2018) Bone turnover markers after the menopause: Tscore approach. Bone 111:44-48

15. Moreira CA, Fitzpatrick LA, Wang Y, Recker RR (2017) Effects of abaloparatide-SC (BA058) on bone histology and histomorphometry: the ACTIVE phase 3 trial. Bone 97:314-319 